\title{
Loss of PR55a promotes proliferation and metastasis by activating MAPK/AKT signaling in hepatocellular carcinoma
}

\author{
JiangSheng Zhao ${ }^{1 \dagger}$, GuoFeng Chen ${ }^{1 \dagger}$, Jingqi Li ${ }^{2}$, Shiqi Liu', Quan Jin ${ }^{1}$, ZhengWei Zhang ${ }^{2}$, Fuzhen Qi ${ }^{1}$, \\ JianHuai Zhang ${ }^{1}$ and JianBo Xu ${ }^{1 *}$ (1)
}

\begin{abstract}
Background: PR55a plays important roles in oncogenesis and progression of numerous malignancies. However, its role in hepatocellular carcinoma (HCC) is unclear. This study aims to characterize the functions of PR55a in HCC.

Methods: PR55a expressions in HCC tissues and paired healthy liver samples were evaluated using Western blot and tissue microarray immunohistochemistry. We knocked down the expression of PR55a in SMMC-7721 and LM3 cell lines via small interfering and lentivirus. In vitro cell counting, colony formation, migration and invasion assays were performed along with in vivo xenograft implantation and lung metastases experiments. The potential mechanisms involving target signal pathways were investigated by RNA-sequencing.

Results: PR55a expression level was suppressed in HCC tissues in comparison to healthy liver samples. Decreased PR55a levels were correlated with poorer prognosis $(P=0.0059)$. Knockdown of PR55a significantly promoted cell proliferation and migration, induced repression of the cell cycle progression and apoptosis in vitro while accelerating in vivo HCC growth and metastasis. Mechanistic analysis indicated that PR55a silencing was involved with MAPK/AKT signal pathway activation and resulted in increased phosphorylation of both AKT and ERK1/2.
\end{abstract}

Conclusions: This study identifies PR55a to be a candidate novel therapeutic target in the treatment of HCC.

Keywords: Hepatocellular carcinoma, PR55a, Cell proliferation, Cancer metastasis, MAPK/AKT signaling pathway

\section{Introduction}

Hepatocellular carcinoma (HCC) represents the seventh most common and the third most fatal malignant tumor [1]. Despite improvements in early diagnostic methods and advanced surgical and medical therapy, HCC carries an abysmally poor prognosis, with less than $10 \%$ of patients surviving more than 5 years from diagnosis [2]. It is therefore of utmost importance

\footnotetext{
*Correspondence: hayyxjb@njmu.edu.cn

†JiangSheng Zhao and GuoFeng Chen contributed equally to this work ${ }^{1}$ Department of Hepatobiliary Surgery, The Affiliated Huaian NO.1

People's Hospital of Nanjing Medical University, Huai'an 223001, Jiangsu, People's Republic of China

Full list of author information is available at the end of the article
}

that improved clinical diagnostic and treatment methods are established.

Inactivation of tumor suppressor genes is a critical mechanism of tumorigenesis. For example, TP53INP1 is significantly down-regulated in liver cancer and promotes metastasis [3]. Protein Phosphatase 2A (PP2A) works with protein kinase to maintain the dynamic balance between states of protein phosphorylation and dephosphorylation. Each PP2A consists of one regulatory $\mathrm{B}$ subunit, one structural subunit (PP2A-A) and one core catalytic subunit (PP2A-C) [4]. Of these, PP2A-A and PP2A-C are considered to be core enzymes and exist as dimeric complexes, while $B$ regulatory subunits exist independently. The PP2A regulatory B subunits can be

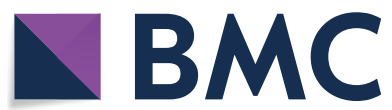

(c) The Author(s) 2021. This article is licensed under a Creative Commons Attribution 4.0 International License, which permits use, sharing, adaptation, distribution and reproduction in any medium or format, as long as you give appropriate credit to the original author(s) and the source, provide a link to the Creative Commons licence, and indicate if changes were made. The images or other third party material in this article are included in the article's Creative Commons licence, unless indicated otherwise in a credit line to the material. If material is not included in the article's Creative Commons licence and your intended use is not permitted by statutory regulation or exceeds the permitted use, you will need to obtain permission directly from the copyright holder. To view a copy of this licence, visit http://creativeco mmons.org/licenses/by/4.0/. The Creative Commons Public Domain Dedication waiver (http://creativecommons.org/publicdomain/ zero/1.0/) applies to the data made available in this article, unless otherwise stated in a credit line to the data. 
classified into PP2A B55/PR55, B56/PR56/PR61, PR48/ PR72/PR130 and PR93/PR110. Isomer molecular weights of these subfamily members are indicated by the designated number with these subfamily numbers indicating the molecular weight of the isomer [5].

Recent studies provide evidence that PR55 $\alpha$ plays important roles in oncogenesis and progression of numerous malignancies. PP2A/PR55 $\alpha$ (PPP2R2A) regulates several crucial pathways that control cell proliferation and metastasis, including the mitogen activated protein kinase (MAPK) and phosphatidylinositol-3-kinase (PI3K)/AKT pathways as well as the c-Myc, YAP, and apoptosis machinery [6, 7]. PP2A may function as either a tumor suppressor $[8,9]$ or oncogene $[10,11]$. PR55 $\alpha$ has been demonstrated to preferentially dephosphorylate phospho-Thr-308 instead of phospho-Ser-473 of the AKT signal pathway in the regulation of cell proliferation and survival of lymphoid cells [12]. In pancreatic ductal carcinoma, elevated PR55 $\alpha$ induces cancer cell proliferation through activation of many oncogenic signaling pathways, including ERK, AKT, and Wnt [13]. In lung non-small cell carcinoma, PR55 $\alpha$ was frequently found at suppressed levels and directly dephosphorylated ATM at S1981, S189 and S367 to promote its presence at double-strand break sites [14].

Our previous study showed that long noncoding RNA GMAN promoted the phosphorylation of eukaryotic translation initiation factor $4 \mathrm{~B}$ (eIF4B) at serine- 422 by preventing the combination of PPP2R2A (PR55 $\alpha$ ) and eIF4B [15]. EIF4B is a key component of translation initiation and its activity is controlled by MAPK and PI3K pathways [16]. PR55 $\alpha$ dephosphorylated eIF4B-Ser422 and repressed mRNA translation and anti-apoptotic protein expression. PR55 $\alpha$ is also reported to negatively regulate the AKT pathway in HCC [17].

This investigation aims to characterize the role of PR55 $\alpha$ as a tumor suppressor in HCC. Its expression appeared to possess a strong correlation with HCC recurrence and poorer prognosis. Physiologically, PR55 $\alpha$ inhibited cell proliferation and metastasis by repressing the MAPK/AKT pathway.

\section{Methods}

\section{Clinical tissues and tissue microarrays}

HCC tissues and matched normal samples from The Affiliated Huai'an NO.1 People's Hospital of Nanjing Medical University were used for western blot. Immunohistochemistry (IHC) analysis was performed on tissue microarray kits comprising of 80 human HCC tissues and 80 adjacent non-tumor tissues that were purchased from Shanghai Outdo Biotech Co. Ltd (HLivH160CS01) (National Human Genetic Resources Sharing Service Platform, Shanghai, China). The diagnosis of HCC was confirmed by pathology. Written informed consent was signed by patients and all experimental protocols were reviewed by the Medical Ethics Committee of Shanghai, the People's Republic of China.

\section{Cell culture and RNA interference by shRNA}

Five HCC cell lines and one normal liver cell line L02 were purchased from KeyGen (Nanjing KeyGen Biotech Co, Ltd, Jiangsu, China). Cells were maintained in $10 \%$ fetal bovine serum(FBS)-supplemented Dulbecco's Minimum Essential Medium (DMEM) along with antibiotics in an atmospheric condition of $5 \% \mathrm{CO}_{2}$ and at 37 ${ }^{\circ} \mathrm{C}$. Three siRNAs were purchased from Gene Pharma (China). PR55 $\alpha$ target sequences utilized in this experiment were the following: GCCUAUGGAUCUAAUGGU UTT for siPR55 $\alpha \# 1$, GCAGAUGAUUUGCGGAUUATT for siPR5 $5 \alpha \# 2$, and GGAAACAUACCAGGUGCAUTT for siPR55 $\alpha \# 3$. Transfection was performed as previously documented [15].

\section{Western blotting}

Western blotting assays were conducted as previously reported [18]. The primary antibodies were as follows PR55 $\alpha$ (1/1000, A2185, Abclonal, China), AKT (1/1000, A17909, Abclonal, China), p-AKT-T308 (1/1000, AP0304, Abclonal, China), P-AKT-S473 (1/1000, AP0140, Abclonal, China), ERK1/2 (1/1000, A4782, Abclonal, China) and p-ERK1/2-T202/Y204 (1/1000, AP0472, Abclonal, China).

\section{Immunohistochemistry (IHC)}

The slices were deparaffinized in xylol, heated for antigen retrieval with sodium citrate $(\mathrm{pH} 6.0)$, and treated by hydrogen peroxide. Subsequently, tissue microarray were incubated overnight with anti-PR $55 \alpha$ at $4{ }^{\circ} \mathrm{C}$. Finally, secondary antibody and DAB chromogentic agent were added. PR $55 \alpha$ staining intensity was quantified based on the following scale: 0 (staining not detectable), 1 (faintly yellow, weak staining), 2 (light brown moderately staining), and 3 (brown, strongly staining). A high PR55 $\alpha$ expression was marked by colour grades of $\geq 2$. The staining results were assessed by independent senior pathologists who remained isolated from each other throughout the experiment.

\section{Cell counting and colony formation assay}

Colony formation assays and Cell Counting Kit-8 (CCK8 ) assays were used to assess the ability of cell proliferation. 96-well plates were used to house transfected HCC cells at a density of $1 \times 10^{3}$ cells per well. After culturing the cells overnight, cells then received a dose of CCK-8 reagent (Dojindo, Shanghai, China). All treatments were administered daily at the same time. Two hours later, a 
microplate reader was used to assess the absorbance values (OD).

Six-well plates were used to house the transfected cells $\left(1 \times 10^{3}\right.$ cells per well) which were then allowed to undergo a 10-day incubation period. Colonies that contained $\geq 50$ cells were selected for counting. Phosphate buffered saline (PBS) was used to rinse the colonies before they were fixed for $30 \mathrm{~min}$ with $4 \%$ paraformaldehyde and exposed for $2 \mathrm{~min}$ to crystal violet. Three individual repeats of each experiment were done.

\section{Cell migration and invasion assay}

Cell invasion and migration were measured by transwell assays. $200 \mu \mathrm{l}$ serum-free medium was used to resuspend $2 \times 10^{4}$ transfected HCC cells before they were placed in the upper cartridge (Millicell, USA) which was precoated with or without $50 \mu \mathrm{l}$ matrigel. The lower cartridge contained $600 \mathrm{ml}$ DMEM medium with 20\% FBS. The cells were then allowed to incubate for $24 \mathrm{~h}$. Paraformaldehyde was then used to fix cells before they were staining using crystal violet. Cells were then photographed and quantified using a microscope for data analysis. The results represented the mean values of three independent experiments.

\section{Cell cycle and apoptosis assay}

Cell cycle and apoptosis assays were conducted using flow cytometer (Beckman Coulter). For cell cycle analysis, transfected cells were harvested and fixed overnight in $70 \%$ ethanol at $4{ }^{\circ} \mathrm{C}$. The cells were then exposed to RNase A before being labeled for 30 min with propidium iodide (PI) at $37^{\circ} \mathrm{C}$.

Cells were collected and stained with Annexin V-FITC and PI reagents prior to the cell apoptosis analysis. Three separate repetitions were carried out for each experiments.

\section{In vivo tumor assay}

$\mathrm{BABL} / \mathrm{c}$ nude mice were obtained from Nanjing Medical University (Nanjing, Jiangsu, China). Six-week-old female nude mice $(n=5)$ were treated over their flanks with subcutaneous injections of approximately $2 \times 10^{6}$ LM3 cells which either possessed stable PR55 $\alpha$ knockdown or PR55 $\alpha$ control. The xenograft tumors were dissected and imaged at 4 weeks post-inoculation. Terminal deoxynucleotidyl transferase (TdT) mediated dUTP nick end labelling (TUNEL) staining and Ki-67 immunofluorescence staining were performed as described previously [15]. For experiments regarding lung metastases, the same cancer cells were administered intravenously into the tail veins of nude mice $(n=5)$. Lung nodules were measured under a dissecting microscope at 40 days after treatment. The Experimental Animal Management
Committee of Nanjing Medical University approved all mice experiments which were formulated in strict compliance to established guidelines.

\section{RNA sequencing}

RNA sequencing analysis and quantification were utilized to assess changes in mRNA profiles of LM3 cells with PR55 $\alpha$ knockdown or PR55 $\alpha$ control. Samples were performed in triplicate. The differentially expressed mRNAs were selected with fold change $<0.5$ or $>2$ and possessed a $\mathrm{P}$ value $<0.05$ by $\mathrm{R}$ package edgeR. We also analyzed GO enrichment and KEGG enrichment in the differentially expressed mRNAs.

\section{Statistical analysis}

Mean \pm standard deviation was used to express all collected data. Analysis was carried out using GraphPadPrism 6. Variances between normal and HCC samples were contrasted using paired t-test. The associations between clinicopathological features and PR55 $\alpha$ expression were assessed by the fishers exact test and Chisquare test. Statistical significance was designated as follows: ${ }^{*} \mathrm{P}<0.05,{ }^{* * *} \mathrm{P}<0.01$, ${ }^{* * *} \mathrm{P}<0.001$ and ${ }^{* * * *} \mathrm{P}<0.001$. $\mathrm{P}<0.05$ was interpreted as a result that was statistically significant.

\section{Results \\ PR55a is downregulated in HCC tissues in contrast to healthy specimens}

To investigate the potential function of PR55 $\alpha$,we assessed the relationship between PR55 $\alpha$ and HCC prognosis. As shown in Fig. 1a, lower PR55 $\alpha$ expression levels were strongly linked to an overall poorer survival $(P=0.0059)$. Likewise, western blotting assays also demonstrated a lower expression of PR55 $\alpha$ in HCC samples (Fig. 1b). We next evaluated PR55 $\alpha$ expression by IHC in a tissue microarray comprising of $80 \mathrm{HCC}$ tissues and adjacent healthy tissues. Our tissue microarray results showed that $57.5 \%(46 / 80)$ HCC tissues demonstrated weak or no PR55 $\alpha$ expression compared with $36.25 \%$ $(29 / 80)$ cases in healthy tissues surrounding the tumor (Fig. 1c, d). IHC analysis found that PR55 $\alpha$ expression in $\mathrm{HCC}$ tissues were significantly lower in comparison to healthy samples $(\mathrm{P}<0.0001)$ (Fig. 1e) (Additional file 1 : Table S1).

In order to explore the potential clinical value of PR55 $\alpha$ expression, the clinical characteristics of $80 \mathrm{HCC}$ patients were analyzed. Correlation analysis of clinical parameters showed that PR55 $\alpha$ expression correlated inversely with TNM stage and vascular invasion (Table 1). 

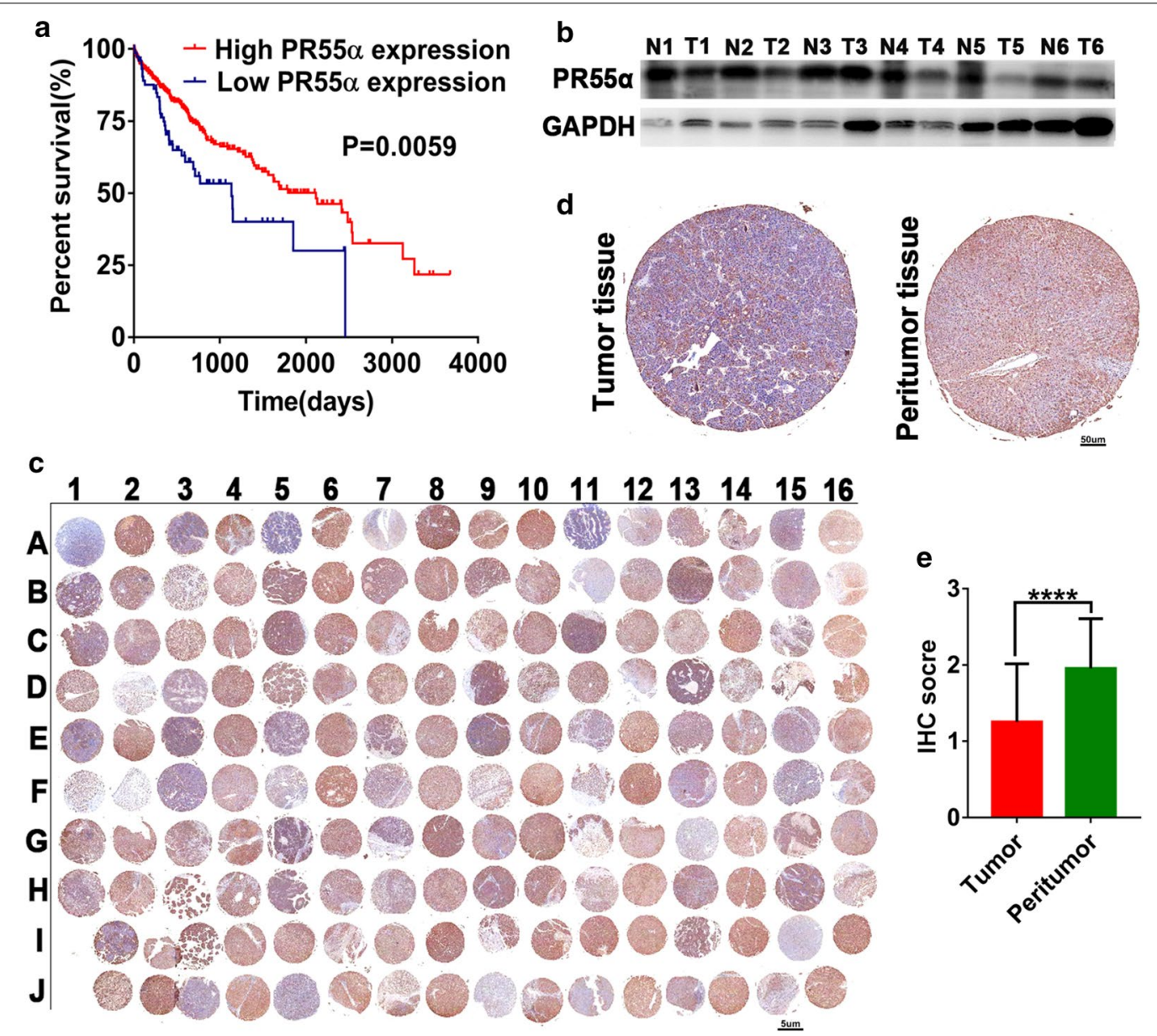

Fig. 1 HCC tissues that expressed lower PR55a levels were noted in patients with poorer prognoses. a The overall survival of patients with either high ( $n=82$ ) or low ( $n=283)$ PR55a expressions (Data extracted from The Human Protein Atlas) (P=0.0059). b PR55a expressions in six paired of HCC tissues and non-adjacent normal liver tissues as determined using Western blotting. c Images of immunohistochemical (IHC) staining of PR55a in 80 paired HCC tumor tissue and peritumor tissue. ( $3 \times$ magnification). The odd rows represent HCC tissues and the even rows represent peritumor tissues. $\mathbf{d}$ Representative IHC-stained images of PR55a in HCC tumor tissue and healthy liver tissues samples. (40x magnification). e IHC scores of PR55a in HCC and matched healthy liver samples $(P<0.0001)$

PR55a knockdown induces HCC cell growth and metastasis In order to fully illustrate the role of PR55 $\alpha$ in HCC cell function, Western blotting experiments were done to quantify HCC cell PR55 $\alpha$ expression levels. As shown in Fig. 2a, PR55 $\alpha$ exhibited a higher expression level in SMMC-7721 and LM3 HCC cell lines as compared to L02 normal liver cells. We then used specific siRNA targeted against PR55 $\alpha$ in LM3 cells and verified the transfection efficiency using immunoblotting assays and real-time PCR (Fig. 2b, c). Stably transfected HCC cell lines were then used for subsequent experiments. Knockdown of PR55 $\alpha$ boosted the migration and invasion ability of both SMMC-772 and LM3 compared with control cells, as evidenced by transwell assays (Fig. 2d). Moreover, colony formation assays and CCK8 assays indicated that PR55 $\alpha$-shRNA significantly promoted proliferation of SMMC-772 and LM3 cells in comparison to control cells (Fig. 2e, f). When interpreted as a whole, we conclude that the migratory and proliferative ability of HCC cells were attenuated by PR55 $\alpha$.

\section{PR55a knockdown suppresses apoptosis and cell cycle arrest in HCC cells}

To further assess the inhibitive function of PR55 $\alpha$, a fluorescence-activated cell sorting (FACS) analysis was done to determine the proportion of SMMC-7721 and LM3 cells in each phase of the cell cycle as well as the percentage that were apoptosed. We discovered that PR55 $\alpha$ knockdown significantly decreased the amount of cells undergoing apoptosis (Fig. 3a) $(\mathrm{P}=0.009$ for SMMC7721 
Table 1 The association between PR55a expression and clinical pathological data in HCC patients

\begin{tabular}{|c|c|c|c|}
\hline \multirow[t]{2}{*}{ Features } & \multicolumn{2}{|l|}{ PR55a level } & \multirow[t]{2}{*}{$\mathbf{P}$} \\
\hline & $\begin{array}{l}\text { High } \\
\text { expression }\end{array}$ & Low expression & \\
\hline Cases & 34 & 46 & \\
\hline \multicolumn{4}{|l|}{ Age (years) } \\
\hline$<60$ & 18 & 30 & \multirow[t]{2}{*}{0.268} \\
\hline$\geq 60$ & 16 & 16 & \\
\hline \multicolumn{4}{|l|}{ Gender } \\
\hline Man & 30 & 38 & \multirow[t]{2}{*}{0.544} \\
\hline Female & 4 & 8 & \\
\hline \multicolumn{4}{|l|}{ Cirrhosis } \\
\hline Yes & 25 & 36 & \multirow[t]{2}{*}{0.791} \\
\hline No & 9 & 10 & \\
\hline \multicolumn{4}{|l|}{ Size $(\mathrm{cm})$} \\
\hline$<5$ & 5 & 5 & \multirow[t]{2}{*}{0.736} \\
\hline$\geq 5$ & 29 & 41 & \\
\hline \multicolumn{4}{|l|}{ TNM } \\
\hline$|-| \mid$ & 18 & 12 & \multirow[t]{2}{*}{0.020} \\
\hline III & 16 & 34 & \\
\hline \multicolumn{4}{|c|}{ Microvascular invasion } \\
\hline Yes & 5 & 24 & \multirow[t]{2}{*}{$0.010^{*}$} \\
\hline No & 29 & 22 & \\
\hline \multicolumn{4}{|c|}{ Histologic grade } \\
\hline Low & 3 & 4 & \multirow[t]{3}{*}{0.890} \\
\hline Middle & 17 & 22 & \\
\hline High & 14 & 20 & \\
\hline
\end{tabular}

${ }^{* P}<00.05$

and $\mathrm{P}=0.0002$ for LM3). As illustrated in Fig. 3b, PR55 $\alpha-$ shRNA induced significantly decreased cells in the G0/1 phase while increasing the number of cells in the $S$ phase $(\mathrm{P}<0.05)$.

\section{Decrease of PR55a promotes cell growth and metastasis in vivo}

We next evaluated the inhibitive function of PR55 $\alpha$ on liver cancer growth and metastasis in vivo. Male nude mice were subjected to subcutaneous injections of LM3 cells that stably expressed either PR55 $\alpha$-shRNA or PR55 $\alpha$-control. Our results showed that PR55 $\alpha$ shRNA cells developed into larger tumors than control cells in nude mice (Fig. 4a). The weights of tumor xenografts also indicated that PR55 $\alpha$ knockdown promoted tumor growth compared with the control cells (Fig. 4b) $(P=0.005)$. Xenograft tissues were also subjected to $\mathrm{H} \& \mathrm{E} / \mathrm{ki} 67$ staining $(\mathrm{P}=0.001)$ and TUNEL assays $(\mathrm{P}=0.002)$ (Fig. 4c). As shown in Fig. 4c, shPR55 $\alpha$ tumor cells demonstrated a significantly decreased proportion of apoptotic DNA fragments and elevated Ki-67 index compared with the control group. In addition, more pulmonary metastases were found in the shPR55 $\alpha$ group (Fig. 4d) $(\mathrm{P}=0.004)$. Based on this information, we conclude that the severity of HCC malignancy is inhibited by PR55 $\alpha$ both in vitro and in vivo.

\section{PR55a inhibits HCC progression through MAPK and AKT signaling}

To explore the molecular mechanisms of PR55 $\alpha$ in HCC, a microarray analysis was performed in LM3 cells with PR55 $\alpha$ knockdown and in control cells. The results revealed 166 down-regulated mRNAs and 754 up-regulated mRNAs (Additional file 2: Table S2). We also uncovered various differentially expressed genes (Fig. 5a). Kyoto Encyclopedia of Genes and Genomes (KEGG) pathway analysis of the genes that were found to be upregulated in PR55 $\alpha$ silenced cells suggested a potential association between PR55 $\alpha$ and MAPK signaling pathways (Fig. 5b). Previous investigations have noted that PR55 $\alpha$ was significantly involved in the AKT signaling pathway [17]. Sh-PR55 $\alpha$ notably increased the amount of phosphorylated AKT-S473, AKT-T308 as well as ERK1/2-T202/Y204 in SMMC-7721 and LM3 cells, as evidenced by Western blotting (Fig. 5c). To verify this phenotype, we treated above cells with the PI3K inhibitor LY294002 and the MEK inhibitor U0126. The results showed that LY294002 and U0126 can reverse PR55 $\alpha$ induced alteration of phosphorylated ERK1/2 or AKT (Fig. 5d). Together, these results suggested a vital role for PR55 $\alpha$ in aberrant MAPK and AKT signaling pathway activation in HCC.

\section{Discussion}

PP2A is a serine/threonine phosphatase which possesses a rather controversial role in tumorigenesis. PR55 $\alpha$ (encoded by PPP2R2A) can function as both an oncogene and tumor suppressor in different cancers. Previous studies revealed that knockdown of PR55 $\alpha$ hindered non-small cell lung cancer cellular growth by increasing JUN T239 phosphorylation [18]. However, more studies supported the tumor suppressing role of PR55 $\alpha$. Cancer cell invasion and proliferation have been noted to be suppressed by PR55 $\alpha$ in diffuse large B-cell lymphoma cells [19] and thyroid cancer [20]. Divergences of transcriptional and posttranscriptional regulation of PR55 $\alpha$ were the possible reasons for this discrepancy. High-resolution analyses of somatic copy number confirmed that significant loss-of heterozygosity (LOH) of PPP2R2Acontaining region lead to down-regulation of PPPP2R2A mRNA expression in many human cancers [21]. The percent of samples with decreased PPP2R2A mRNA expression in lung cancer, thyroid gland cancer, breast cancer and HCC were $36.82 \%, 94.44 \%, 65.21 \%$ and 


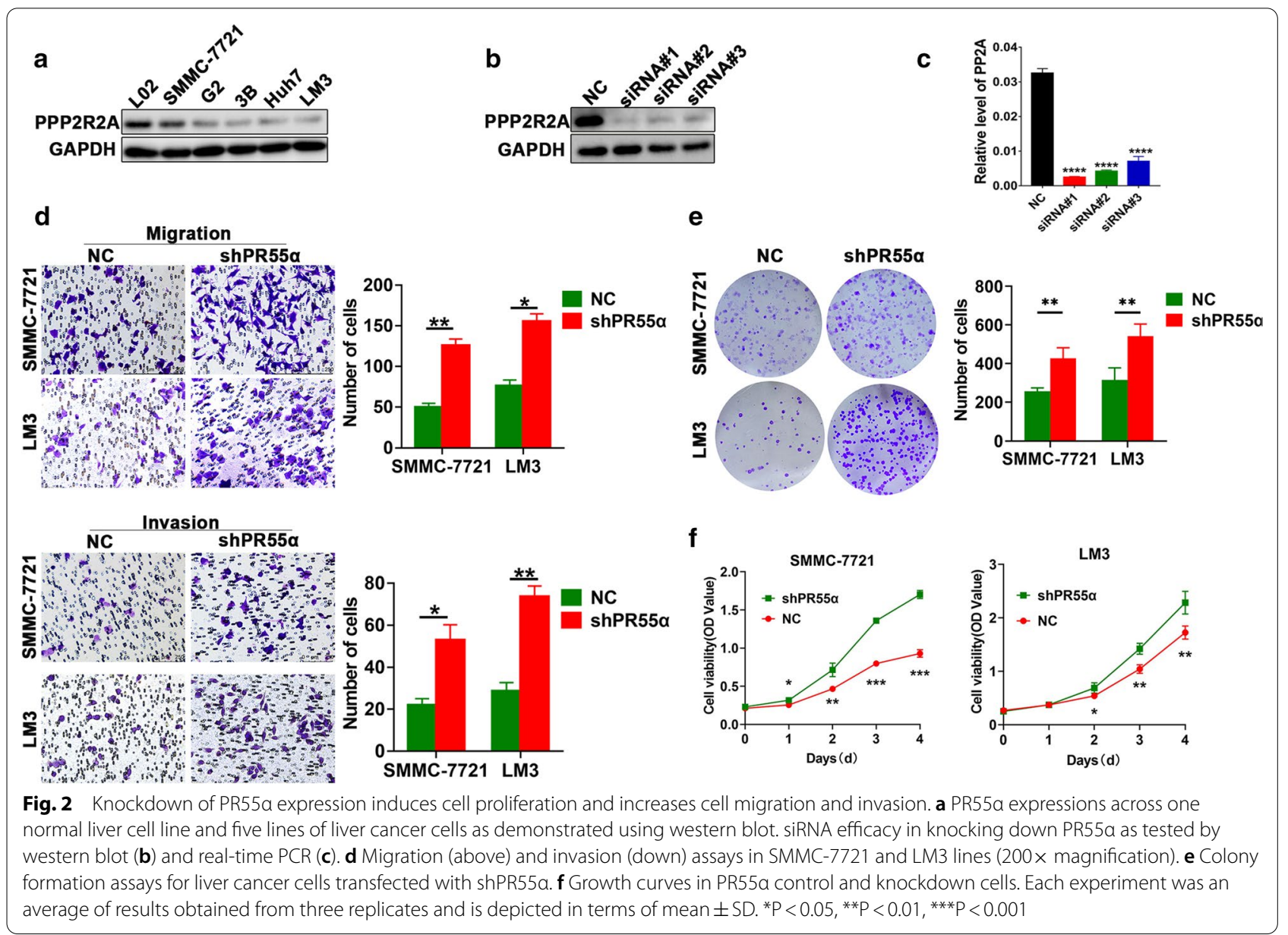

$58.82 \%$ respectively. However, only $20 \%$ of PPP2R $2 A$ mRNA expression was reduced in pancreatic cancer. Additionally, the expression of PPP2R2A is also regulated by multiple MirRNAs. For example, MiR-222 can suppress PPP2R2A expression and promote the proliferation or invasion of papillary thyroid cancer [20], diffuse large B-cell lymphoma [19] and HCC [17]. But, miR-665 was reported to promote PPP2R2A overexpression and inhibit the proliferation and epithelial-mesenchymal transition of gastric cancer cells [22]. Specifically, PR55 $\alpha$ was lowly expressed in lung cancer, thyroid gland cancer, breast cancer, diffuse large B-cell lymphoma and HCC, but highly expressed in pancreatic cancer and gastric cancer. To our knowledge, this is the first study indicating PR55 $\alpha$ expressions were frequently at low expression and acted as a regulator of cell proliferation and metastasis in HCC.

In current research, PR55 $\alpha$ expression was found to be suppressed in HCC tissues, and the negative correlation between PR55 $\alpha$ down regulation, several malignant characteristics and poor prognosis was confirmed.
Functionally, PR55 $\alpha$ knockdown significantly triggered cell proliferation and invasion, induced cessation of cell cycle progression and facilitated both in vivo and in vitro cell apoptosis. Furthermore, using both microarray analysis and western blot, we demonstrated that sh-PR55 $\alpha$ could activate the MAPK/AKT signaling pathway. All these results indicate that PR55 $\alpha$ is a tumor suppressor in HCC and may be a reliable biomarker as well as a means to facilitate earlier HCC diagnosis and more effective treatment.

Phosphatases provide both positive and negative regulation for the MAPK pathway at various points. Previous studies showed that PP2A/PR55 $\alpha$ facilitated ERK1/2 phosphorylation/activation by activating KSR and Raf [10, 23]. However, PR55 $\alpha$ has been reported to negatively regulate RAS signaling [24]. PR55 $\alpha$ has been reported to inhibit phosphorylation of ERK1/2 in non-small cell lung cancer cells [14] and vascular smooth muscle cells [25]. Our studies showed that knockdown of PR55 $\alpha$ significantly induced MAPK signaling. Consistent with the microarray analysis, 

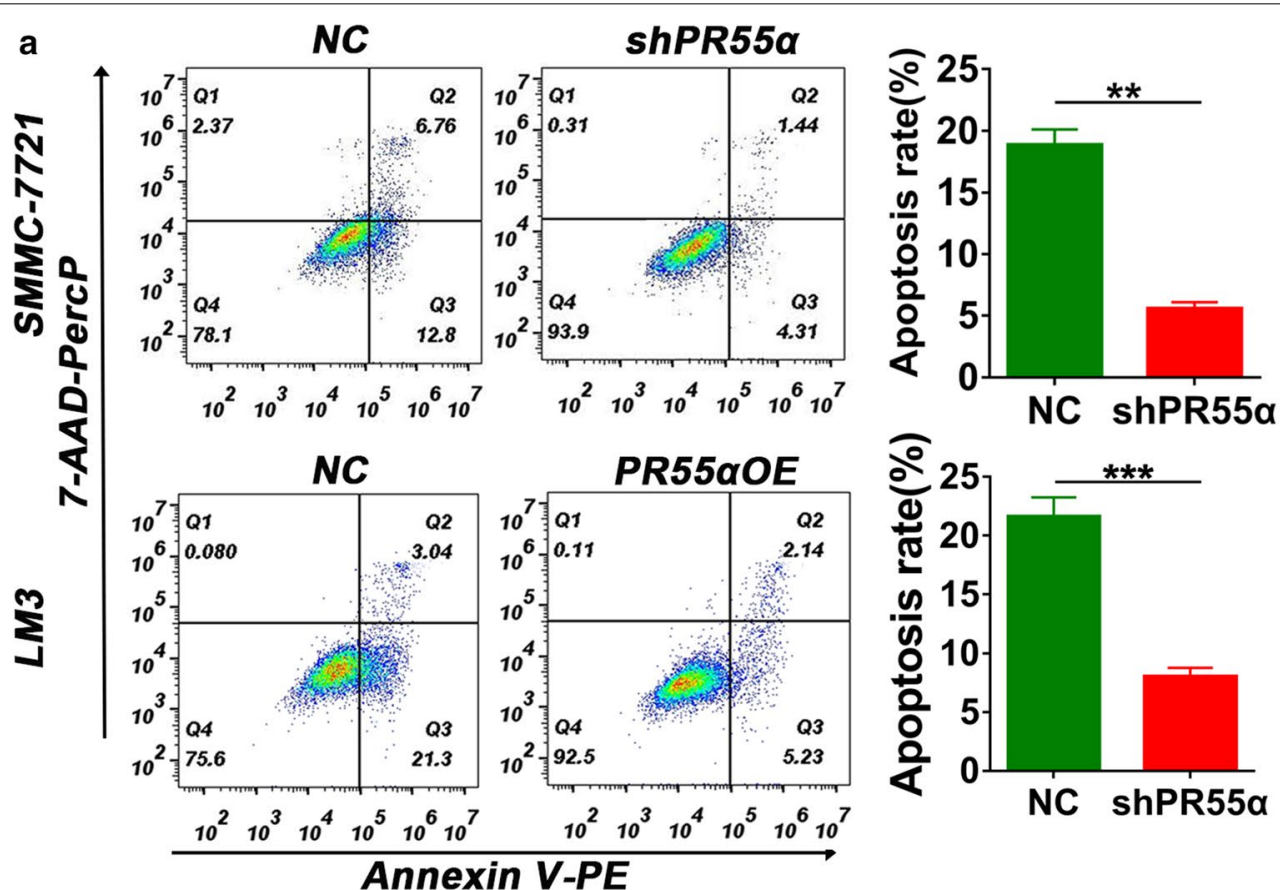

b

NC

shPR55a
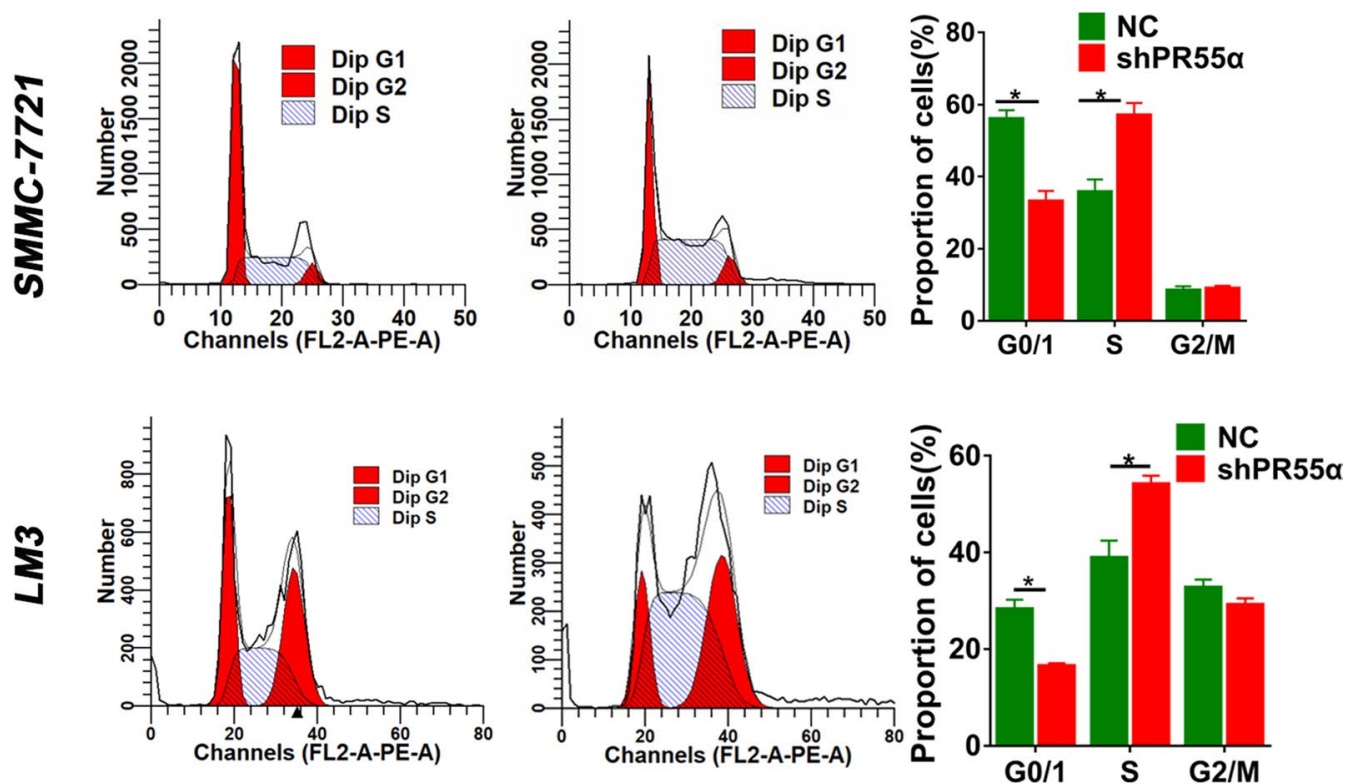

Fig. 3 PR55a silencing represses cell cycle arrest and apoptosis. a Downregulation of PR55a expression inhibits apoptosis in SMMC-7721 and LM3 cells in contrast to the NC group $(P<0.05)$. $\mathbf{b}$ The cell cycles of LM3 and SMMC-7721 cells upon transfection with shPR55a and NC were analyzed using a flow cytometer $(P<0.05)$

western blot showed that shPR55 $\alpha$ promoted Erk1/2 phosphorylation.

In HCC, PI3K/AKT pathway activation represents a significant oncogenic process that has been documented to be commonly activated in HCC [26]. Complete activation of $\mathrm{AKT}$ required phosphorylation at Ser473 by mTORC 2 and at Thr308 by PDK 1 [27, 28]. AKT is activated by various kinases, such as PKA, ACK1 and 


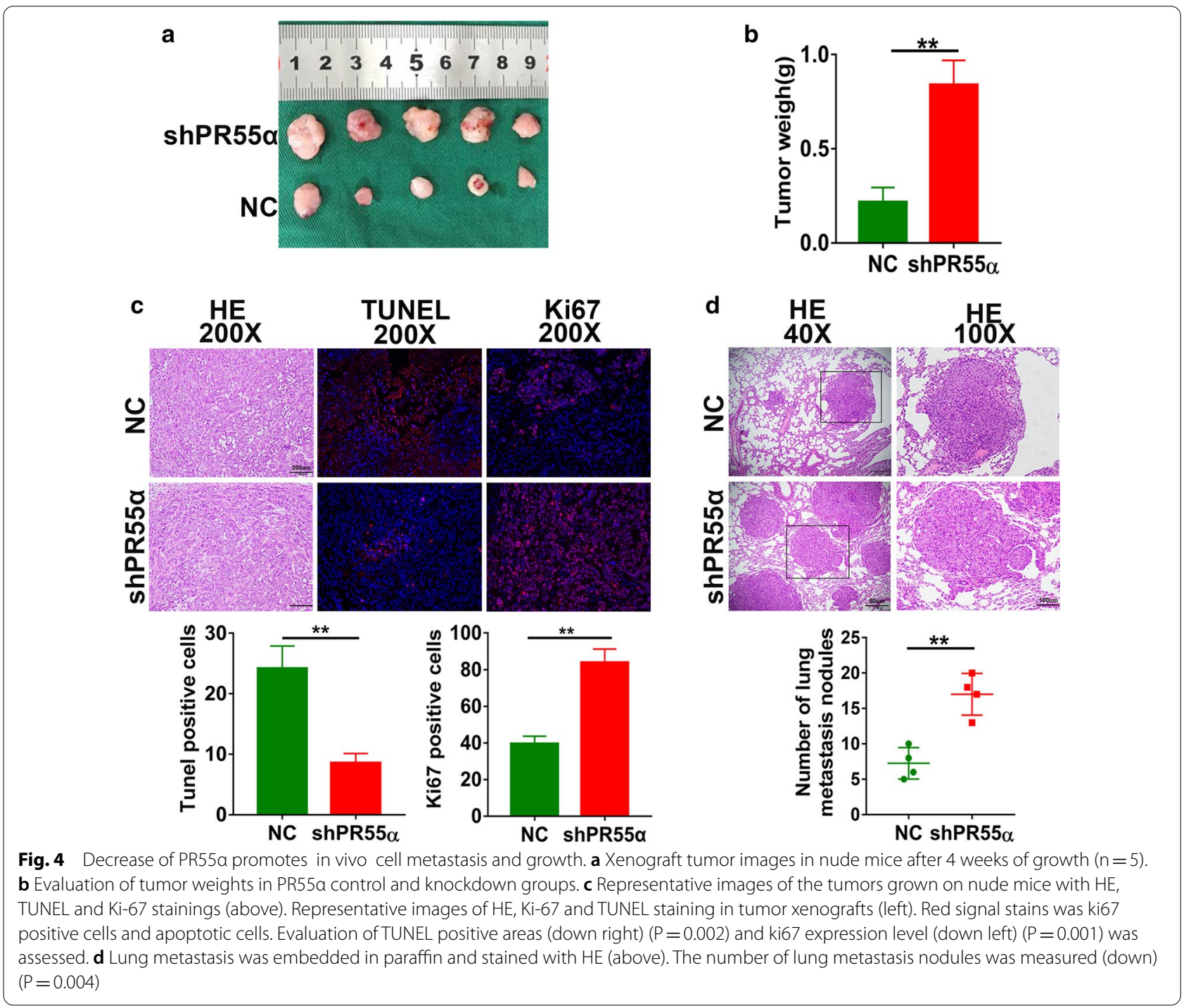

TNK2, and is inhibited by a variety of phosphatases, such as PP2A, PTEN, PHLPPs and INPP4B $[6,29]$. A previous study confirmed that PR55 $\alpha$ can directly bind and induce preferential dephosphorylation of phospho-Thr-308 instead of phospho-Ser-473, as evidenced by in vitro assays on dephosphorylation using both NIH3T3 and FL5.12 cells [30]. However, PR55 $\alpha$ silencing increased baseline phosphorylation of AKT-Thr308 and inhibited Akt-Ser473 phosphorylation upon exposure to insulin-like growth factor-1 in H9c2 cells [31]. More importantly, PR55 $\alpha$ significantly regulated AKT phosphorylation at the Thr-308 and Ser-473 residues in pancreatic cancer cells [13] and liver cancer cells [17]. The discrepancy among these previous findings may be due to the differences in cell type or tumor type. Here, our results suggest that decreased PR55 $\alpha$ expression is responsible for increased AKT-Ser473 and AKT-Thr308 phosphorylation in HCC.

Some limitations need to be pointed out here. First, we failed to analyze the effect of PR55 $\alpha$ on OS and PFS after surgery due to the lack of follow-up data on tissue microarray. Second, PP2A comprises of PP2A-A, PP2A-B and PP2A-C. But, we did not detect the expression of other subunits of PP2A in HCC in this article. Lastly, previous studies showed PR55 $\alpha$ can regulate multiple oncogenic signaling pathways via dephosphorylation of key gene sites. In this article, only transcriptome sequencing may not fully reveal the potential carcinogenic effects of PR55 $\alpha$ in HCC. All these questions need further study to investigate. In the next step, phosphorylation analysis with or without PR55 $\alpha$ knockdown may provide a better understanding of its underlying mechanisms of action and phosphorylation sites. 


\section{a}

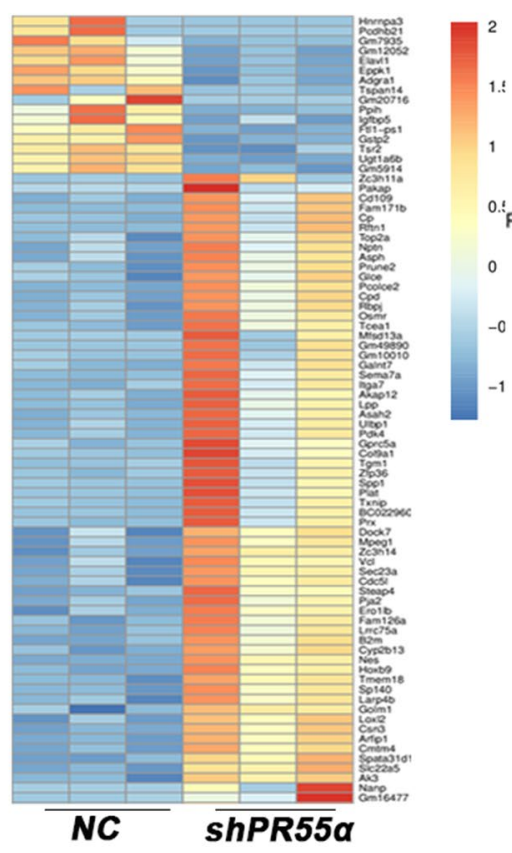

c

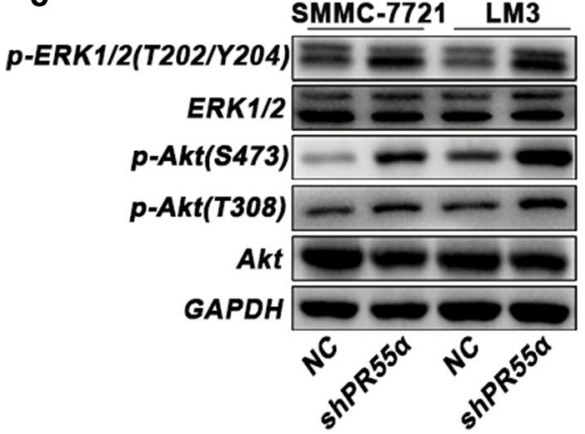

0 b

Fluid shear stress and atherosclerosis MAPK signaling pathway Toll-like receptor signaling pathway Salmonella infection

ECM-receptor interaction

Parathyroid hormone synthesis, secretion and action

Hypertrophic cardiomyopathy ( $\mathrm{HCM})$

Ferroptosis

Pertussis

Small cell lung cancer Regulation of actin cyloskeleton Estrogen signaling pathway Hedgehog signaling pathway Fanconi anemia pathway Influenza A

Protein processing in endoplasmic reticulum. Complement and coagulation cascades TNF signaling pathway Toxoplasmosis Legionellosis
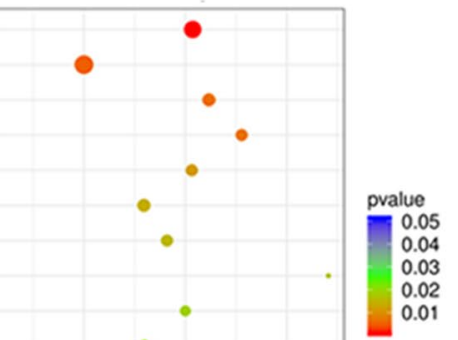

d

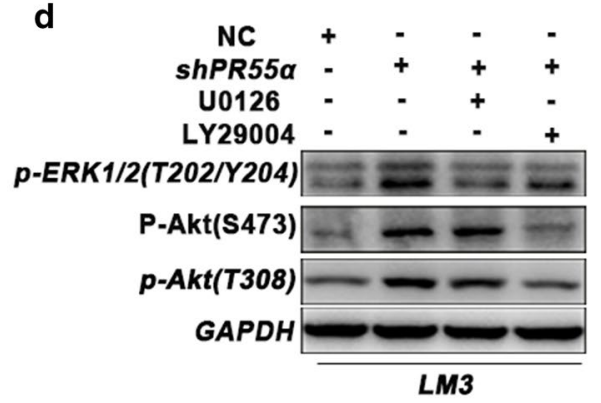

Fig. 5 Knockdown of PR55a by shRNA activates AKT and ERK1/2 signaling. a Microarray analysis was conducted to screen the mRNAs which were subjected to PR55a regulation. b KEGG pathway analysis revealed the potential signaling pathways involved in PR55a. c AKT/ERK signaling pathway protein expressions in HCC cells transfected with shPR55a or shControl were evaluated using western blot. $\mathbf{d}$ The indicated proteins of AKT/ERK

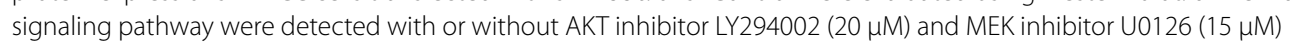

\section{Conclusion}

In conclusion, our results highlight a potential prognostic value of PR55 $\alpha$, and PR55 $\alpha$ inhibits proliferation and metastasis of HCC cells likely by inactivating MAPK/ AKT signaling.

\section{Supplementary Information}

The online version contains supplementary material available at https://doi. org/10.1186/s12935-021-01796-0.

Additional file 1: Table S1. IHC score of PR55a expression in HCC tissues and paired healthy samples.
Additional file 2: Table S2. The results of microarray analysis showed 166 down-regulated mRNAs and 754 up-regulated mRNAs.

\section{Acknowledgements}

We sincerely appreciate all laboratory members.

\section{Authors' contributions}

XJB and CGF designed and wrote this manuscript. ZJS, LJQ, LSQ, JQ and ZWG performed experiments. ZJH and QFZ guide the experiment methods. All authors read and approved the final manuscript.

\section{Funding}

This work was supported by the Fundamental Research Funds for the Nanjing Medical University (NMUB2019340) and the Fundamental Research Funds for The Affiliated Huaian NO.1 People's Hospital of Nanjing Medical University (YLCT202003; YGRS202004). 


\section{Availability of data and materials}

Related data and materials could be seen in the manuscript and the additional files.

\section{Ethics approval and consent to participate}

All 11 numbers of the Hospital's Protection of Human Subjects Committee approved our present study.

\section{Consent for publication}

Written informed consent for publication was obtained from all participants.

\section{Competing interests}

The authors declare no conflict of interest.

\section{Author details}

${ }^{1}$ Department of Hepatobiliary Surgery, The Affiliated Huaian NO.1 People's Hospital of Nanjing Medical University, Huai'an 223001, Jiangsu, People's Republic of China. ${ }^{2}$ Department of Pathology, The Affiliated Huaian NO.1 People's Hospital of Nanjing Medical University, Huai'an 223001, Jiangsu, People's Republic of China.

Received: 29 December 2020 Accepted: 27 January 2021

Published online: 15 February 2021

\section{References}

1. Bray F, Ferlay J, Soerjomataram I, Siegel RL, Torre LA, Jemal A. Global cancer statistics 2018: GLOBOCAN estimates of incidence and mortality worldwide for 36 cancers in 185 countries. CA Cancer J Clin. 2018:68:394-424

2. Bruix J, Reig M, Sherman M. Evidence-based diagnosis, staging, and treatment of patients with hepatocellular carcinoma. Gastroenterology. 2016;150:835-53.

3. Ng KY, Chan LH, Chai S, Tong M, Guan XY, Lee NP, Yuan Y, Xie D, Lee TK, Dusetti NJ, Carrier A, Ma S. TP53INP1 downregulation activates a p73-dependent DUSP10/ERK signaling pathway to promote metastasis of hepatocellular carcinoma. Cancer Res. 2017;77:4602-12.

4. Seshacharyulu P, Pandey P, Datta K, Batra SK, Phosphatase. PP2A structural importance, regulation and its aberrant expression in cancer. Cancer Lett. 2013;335:9-18.

5. Eichhorn PJ, Creyghton MP, Bernards R. Protein phosphatase 2A regulatory subunits and cancer. Biochim Biophys Acta. 2009;1795:1-15.

6. Narla G, Sangodkar J, Ryder CB. The impact of phosphatases on proliferative and survival signaling in cancer. Cell Mol Life Sci. 2018;75:2695-718.

7. Hein AL, Brandquist ND, Ouellette CY, Seshacharyulu P, Enke CA, Ouellette MM, Batra SK, Yan Y. PR55!'Á regulatory subunit of PP2A inhibits the MOB1/LATS cascade and activates YAP in pancreatic cance $r$ cells. Oncogenesis. 2019;8:63

8. Moreno CS, Ramachandran S, Ashby DG, Laycock N, Plattner CA, Chen W, Hahn WC, Pallas DC. Signaling and transcriptional changes critical for transformation of human cells by simian virus $40 \mathrm{~s}$ mall tumor antigen or protein phosphatase 2A B56gamma knockdown. Cancer Res. 2004;64:6978-88

9. Xiao G, Chan LN, Klemm L, Braas D, Chen Z, Geng H, Zhang OC, Aghajanirefah A, Cosgun KN, Sadras T, Lee J, Mirzapoiazova T, Salgia R, Ernst T, Hochhaus A, Jumaa H, Jiang X, Weinstock DM, Graeber TG, $M^{-1}{ }^{1}$ schen $M$. B-cell-specific diversion of glucose carbon utilization reveals a unique vulnerability in B cell Mali gnancies. Cell. 2018;173:470-84.e418.

10. Ory S, Zhou M, Conrads TP, Veenstra TD, Morrison DK. Protein phosphatase 2A positively regulates Ras signaling by dephosphorylating KSR1 and Raf-1 on crit ical 14-3-3 binding sites. Curr Biol. 2003;13:1356-64.

11. Bajpai R, Makhijani K, Rao PR, Shashidhara LS. Drosophila Twins regulates Armadillo levels in response to Wg/Wnt signal. Development. 2004;131:1007-16.

12. Kuo YC, Huang KY, Yang CH, Yang YS, Lee WY, Chiang CW. Regulation of phosphorylation of Thr-308 of Akt, cell proliferation, and survival by the B55alpha regulatory subunit targeting of the protein phosphatase $2 \mathrm{~A}$ holoenzyme to Akt. J Biol Chem. 2008;283:1882-92.

13. Hein AL, Seshacharyulu P, Rachagani S, Sheinin YM, Ouellette MM, Ponnusamy MP, Mumby MC, Batra SK, Yan Y. PR55alpha subunit of protein phosphatase 2A supports the tumorigenic and metastatic potential of pancreatic cancer cells by sustaining hyperactive oncogenic signaling. Cancer Res. 2016:76:2243-53.

14. Shen S, Yue H, Li Y, Qin J, Li K, Liu Y, Wang J. Upregulation of miR-136 in human non-small cell lung cancer cells promotes Erk1/2 activation by targe ting PPP2R2A. Tumour Biol. 2014;35:631-40.

15. Xu J, Lu Y, Liu Q, Xia A, Zhao J, Xu X, Sun Q, Qi F, Sun B. Long noncoding RNA GMAN promotes hepatocellular carcinoma progression by interacting with elF4B. Cancer Lett. 2020:473:1-12.

16. Shahbazian D, Roux PP, Mieulet V, Cohen MS, Raught B, Taunton J, Hershey JW, Blenis J, Pende M, Sonenberg N. The mTOR/PI3K and MAPK pathways converge on elF4B to control its phosphorylation and activity. EMBO J. 2006;25:2781-91.

17. Wong QW, Ching AK, Chan AW, Choy KW, To KF, Lai PB, Wong N. MiR222 overexpression confers cell migratory advantages in hepatocellular carcinoma through enhancin g AKT signaling. Clin Cancer Res. 2010;16:867-75.

18. Cui R, Jiang N, Zhang M, Du S, Ou H, Ge R, Ma D, Zhang J. AMOTL2 inhibits JUN Thr239 dephosphorylation by binding PPP2R2A to suppress the proliferation in non- small cell lung cancer cells. Biochim Biophys Acta Mol Cell Res. 2020;1868:118858.

19. Sun S, Wang H, Ji M. Overexpression of miR-222-3p promotes the proliferation and inhibits the apoptosis of diffuse large B-cell lym phoma cells via suppressing PPP2R2A. Technol Cancer Res Treat. 2019:18:1533033819892256.

20. Huang Y, Yu S, Cao S, Yin Y, Hong S, Guan H, Li Y, Xiao H. MicroRNA-222 promotes invasion and metastasis of papillary thyroid cancer through targeting protein $\mathrm{P}$ hosphatase 2 regulatory subunit B alpha expression. Thyroid. 2018:28:1162-73.

21. Kalev P, Simicek M, Vazquez I, Munck S, Chen L, Soin T, Danda N, Chen W, Sablina A. Loss of PPP2R2A inhibits homologous recombination DNA repair and predicts tumor sensitivity to PARP inhibition. Cancer Res. 2012;72:6414-24.

22. Zhang M, Wang S, Yi A, Qiao Y. microRNA-665 is down-regulated in gastric cancer and inhibits proliferation, invasion, and EMT by tar geting PPP2R2A. Cell Biochem Funct. 2020:38:409-18.

23. Adams DG, Coffee RL, Zhang H, Pelech S, Strack S, Wadzinski BE. Positive regulation of Raf1-MEK1/2-ERK1/2 signaling by protein serine/threonine phosphatase 2A holoen zymes. J Biol Chem. 2005;280:42644-54.

24. Kauko O, Westermarck J. Non-genomic mechanisms of protein phosphatase 2A (PP2A) regulation in cancer. Int J Biochem Cell Biol. 2018;96:157-64.

25. Zhang CF, Kang K, Li XM, Xie BD. MicroRNA-136 promotes vascular muscle cell proliferation through the ERK1/2 pathway by targeting PPP2 R2A in atherosclerosis. Curr Vasc Pharmacol. 2015;13:405-12.

26. Li W, Tan D, Zhang Z, Liang JJ, Brown RE. Activation of Akt-mTOR-p70S6K pathway in angiogenesis in hepatocellular carcinoma. Oncol Rep. 2008;20:713-9.

27. Faes S, Dormond O. PI3K and AKT: unfaithful partners in cancer. Int J Mol Sci. 2015;16:21138-52.

28. Fruman DA, Chiu H, Hopkins BD, Bagrodia S, Cantley LC, Abraham RT. The PI3K pathway in human disease. Cell. 2017;170:605-35.

29. Xia Q, Ali S, Liu L, Li Y, Liu X, Zhang L, Dong L. Role of ubiquitination in PTEN cellular homeostasis and its implications in GB drug resistance. Front Oncol. 2020;10:1569.

30. Kuo YC, Huang KY, Yang CH, Yang YS, Lee WY, Chiang CW. Regulation of phosphorylation of Thr-308 of Akt, cell proliferation, and survival by the B55alpha reg ulatory subunit targeting of the protein phosphatase 2A holoenzyme to Akt. J Biol Chem. 2008;283:1882-92.

31. Tobisawa T, Yano T, Tanno M, Miki T, Kuno A, Kimura Y, Ishikawa S, Kouzu $\mathrm{H}$, Nishizawa K, Yoshida H, Miura T. Insufficient activation of Akt upon reperfusion because of its novel modification by reduced PP2A-B55 !Á contributes to enlargement of infarct size by chronic kidney disease. Basic Res Cardiol. 2017:112:31.

\section{Publisher's note}

Springer Nature remains neutral with regard to jurisdictional claims in published maps and institutional affiliations. 